\title{
¿Quién es el trol??: la construcción de identidades políticas en social media en el contexto peruano
}

\author{
Diego Cerna Aragón \\ Instituto de Estudios Peruanos \\ dcerna@iep.org.pe
}

Recibido: 1/9/2017 / Aceptado: 3/10/2017

doi: 10.26439/contratexto2017.n028.1551

\begin{abstract}
Resumen. En social media es común encontrar acusaciones descalificadoras entre quienes pertenecen a grupos políticos $\mathrm{u}$ orientaciones ideológicas distintas. El objetivo del artículo es afirmar que estas prácticas no son accesorias o superfluas, sino que son constitutivas en la formación y consolidación de las identidades políticas de quienes participan en estas discusiones. Para ello, se revisa literatura académica actual sobre tres tendencias recientes en las dinámicas de discusión política en social media (la polarización en el debate político, la concentración en personalidades y figuras, y las prácticas de los troles) y, utilizando herramientas tomadas de la netnografía, se exploran y aplican estos conceptos al contexto peruano. Al hacer esto, el artículo ofrece un bosquejo sobre la dinámica política cotidiana en social media, sobre cómo se forman bandos y ocurren disputas, y cómo esto sirve para la (re)producción de identidades políticas. Los resultados de la exploración muestran un escenario donde predomina la polarización entre distintas posiciones políticas y la incertidumbre sobre la información y las identidades que circulan.
\end{abstract}

Palabras clave: social media / troles / comunidades virtuales / identidad política / Perú 


\title{
Who is the Troll?: The Construction of Political Identities on Social Media in the Peruvian Context
}

\begin{abstract}
Авstract. One can easily find discrediting accusations between users with different political affiliations or ideological orientations on social media. The objective of this article is to affirm that these practices are neither superfluous nor secondary, but rather fundamental in the formation and consolidation of political identities of those who participate in these discussions. This article reviews up-to-date academic literature about three recent trends in the dynamics of political discussion on social media (the polarization of the political debate, the focus on personalities and figures, and the trolls' practices) and, using tools taken from Netnography, it explores and applies these concepts to the Peruvian context. Consequently, the article offers an outline about the daily political dynamics on social media, how users take sides and disputes happen, and how this serves to the (re)production of political identities. The results of this investigation show a scenario where two characteristics prevail: the polarization between different political stances and the uncertainty about the information and identities in circulation.
\end{abstract}

Keywords: social media / trolls / virtual communities / political identity / Peru 


\section{Introducción}

F

ujitrolls ${ }^{1}$ y caviares $^{2}$. Estos, entre otros, son términos comunes en las discusiones políticas entre peruanos en social media. Ambos calificativos pueden ser vistos como simples intentos de descalificación de oponentes políticos; no obstante, su misma enunciación puede ser considerada una práctica que (re)produce la identidad de los enunciadores.

En cuanto a su utilidad política, las plataformas de social media, como Facebook y Twitter, son frecuentemente tratadas como tecnologías que facilitan o propician eventos trascendentes, como movilizaciones o acciones de activistas (Della Porta, 2011; Villanueva, 2015). Por otro lado, fuera de estos acontecimientos, la utilidad política de estas plataformas suele ser reducida a "usos cívicos", como un medio que empodera a ciudadanos frente a actores políticos tradicionales, facilitando su asociación (Jensen, Danziger y Venkatesh, 2007; Villanueva, 2015).
Por otro lado, la mirada a la cotidianidad en internet suele estar enfocada en las comunidades que se conforman alrededor de distintas aficiones de la cultura pop (fandom), como series o música, y las celebridades que las crean o interpretan. Trabajos como los de Jenkins (2008) han marcado la pauta sobre cómo estas comunidades son usualmente observadas, poniendo especial atención en los contenidos que generan los propios usuarios.

El objetivo del presente artículo es ofrecer un bosquejo sobre la cotidianidad de las discusiones políticas en social media, sobre cómo se constituyen bandos y ocurren disputas, y cómo estas prácticas forman y mantienen las identidades políticas en el contexto peruano. El artículo está dividido en seis partes. En primer lugar, se describe cómo es que las discusiones políticas en social media suelen producir una mayor polarización en lugar de un debate alturado que conduzca a un consenso. En segundo lugar, se trata cómo es que

1 El término fujitroll se refiere a una persona, específicamente a un usuario de alguna plataforma de social media, que ataca a los oponentes políticos del fujimorismo, ya sea a cambio de beneficios materiales o por una real convicción ideológica. De manera similar, otros términos con el sufijo -troll han sido creados para señalar a otros usuarios, dependiendo de a quienes ataquen en social media.

2 El término caviar se refiere a una persona con una orientación política progresista y que tiene un nivel de vida acomodado (Meléndez, 2012). En este sentido, el término trata de señalar una supuesta contradicción entre su orientación política y su posición social. Debido a esta contradicción, quienes son calificados de caviares son consideradas personas alejadas de la realidad social, superficiales e, incluso, hipócritas. A diferencia de los términos que tienen el sufijo -troll, la palabra caviar no surge en plataformas de social media, ni su uso se limita a estas, aunque gracias a estas plataformas se ha extendido entre los hablantes. 
las características de las plataformas de social media propician el surgimiento de personalidades y figuras que son objeto de aclamación o rechazo. En tercer lugar, se revisa cómo se define a un trol, cuáles son sus prácticas y cómo es que calificar a otros como troles puede servir como una forma de castigo. En cuarto lugar, se detalla la metodología empleada para la exploración. Posteriormente, se discute cómo los conceptos anteriores se encuentran en la cotidianidad de la política peruana en social media. Por último, se plantean algunas conclusiones y recomendaciones sobre cómo manejarse en contextos de polarización e incertidumbre, así como posibles líneas de trabajo que abre este artículo.

\section{Polarización en social media}

Las llamadas nuevas tecnologías han causado cambios importantes en la forma en que las personas hablan sobre política. Tendencias como el reforzamiento en el cambio de énfasis de información a entretenimiento y opiniones, el desvanecimiento de la distinción entre medios convencionales y alternativos, y la atención cada vez mayor a públicos específicos (Shah et al., 2017) han marcado los escenarios de la comunicación en los últimos años, incluyendo la comunicación política. Si bien los espacios producidos por estas nuevas tecnologías, como las plataformas de social media, abrieron la posibilidad de acceder a una diversidad de opiniones y a participar en debates de temas trascendentales, estos frecuentemente se utilizan para buscar contenidos que refuercen las posiciones políticas (Shah et al., 2017), y para comportarse de manera agresiva al comunicarse (Hutchens, Cicchirillo y Hmielowski, 2015).

Las discusiones políticas en social media frecuentemente terminan desembocando en la oposición a posiciones políticas contrarias antes que en un debate sobre hechos o datos (Hutchens et al., 2015). Más aún, cuando se discuten hechos y datos, estos difícilmente hacen variar las posturas iniciales de las partes involucradas, e incluso pueden llegar a reforzarlas (Nyhan y Reifler, 2010). Ciertamente, la creencia o el escepticismo respecto a hechos y datos en discusiones políticas se ven influenciados en mayor medida por factores como la afiliación política y la identificación social con la problemática, y la exposición a información sobre temas controversiales puede incrementar la polarización en lugar de crear acercamientos (Hart y Nisbet, 2011).

No obstante, más allá de la homofilia presente en las interacciones en social media, esto no implica que la información esté absolutamente constreñida en cámaras de eco. Por el contrario, en ocasiones, la información permea gran parte o la totalidad de la red (Barberá, Jost, Nagler, Tucker y Bonneau, 2015). De esta manera, de acuerdo con Garret, Weeks y Neo (2016), la polarización no se produce por un déficit de información de alguna o todas las partes enfrentadas, 
dado que no existiría un filtro que aísle a los individuos de contenidos contrarios a sus posiciones o creencias, como sugiere Pariser (2011), sino que se produciría por el grado de influencia que tienen los medios con contenidos ideológicamente sesgados en las personas, más allá de si están expuestas a perspectivas distintas. Debido a la capacidad de crear y distribuir contenido en internet, los usuarios pueden encontrar contenidos que apoyen casi cualquier creencia o ideología posible (Garret et al., 2016).

En este sentido, existen dos factores importantes para entender el proceso de polarización en social media: la exposición selectiva y el sesgo de confirmación. Los usuarios seleccionan información que se adhiere a su punto de vista, reforzándolo, y no toman como válida la información que no es acorde a él (Del Vicario, Zollo, Caldarelli, Scala y Quattrociocchi, 2016). Esta dinámica produce que los intentos por corregir información en discusiones políticas en social media sean poco efectivos y que se terminen configurando comunidades polarizadas que interactúan entre ellas (Del Vicario et al., 2016; Bessi et al., 2016). El choque de narrativas conflictivas de comunidades enfrentadas, finalmente, provoca que los usuarios por lo general terminen adhiriéndose a alguna de estas comunidades, lo cual puede tener como consecuencia última la reducción de la heterogeneidad de puntos de vista, considerada un componente vital para la democracia de las sociedades (Bessi et al., 2016). Incluso en el caso de discusiones alrededor de acontecimientos no políticos, la polarización suele ocurrir conforme la discusión va adquiriendo matices políticos (Barberá et al., 2015). A la postre, esta polarización por grupos puede llevar eventualmente a un clima de polarización mayor en toda la sociedad (Strandberg, Himmelroos y Grönlund, 2017).

\section{Personalización, celebridad $y$ "malos objetos"}

La personalización en la política no es un fenómeno reciente. Desde hace décadas, otros medios, especialmente la televisión, ya habían iniciado este fenómeno. En este sentido, más que un nuevo fenómeno, lo que se observa en social media es una tendencia en el tiempo (Van Aelst, Sheafer y Stanyer, 2012) o una variación en el grado de énfasis (McGregor, Lawrence y Cardona, 2016). De acuerdo con Van Aelst et al. (2012), la personalización tiene dos dimensiones. Por un lado, se encuentra la individualización, la cual se refiere a la mayor visibilidad de políticos individuales en lugar de partidos, o de miembros individuales de un gabinete en lugar del gobierno. La otra dimensión de la individualización es la privatización, que se refiere a la mayor atención de los medios al aspecto privado de la vida de un político en lugar de su rol público. Si bien la descripción de ambas dimen- 
siones resaltan cómo es que los medios llevan a cabo la personalización en la política, no debe perderse de vista que los mismos políticos también colaboran en este proceso. En otras palabras, la personalización no es algo que solo los medios "le hacen" a la política, sino que los políticos también "se lo hacen a ellos mismos" (McGregor et al., 2016, p. 266).

Dentro de esta tendencia, las plataformas de social media son especialmente relevantes, dado que permiten a los políticos compartir fácilmente información sobre su vida privada o sus intereses y experiencias personales (Graham, Jackson y Broersma, en prensa). Participar en social media requiere construir una representación de uno mismo, lo que les abre la posibilidad a los políticos de (re)presentarse a sí mismos como personas agradables (McGregor et al., 2016). Las plataformas de social media les dan la oportunidad de establecer estrategias comunicativas con el objetivo de reducir la desconexión entre ellos y quienes dicen representar (Graham et al., en prensa). De esta manera, las comunicaciones de carácter personal tratan de acortar la distancia psicológica entre los políticos y los ciudadanos (Hermans y Vergeer, 2012), así como de crear "un sentido de intimidad (imaginada) y cercanía (emocional)", intentando transmitir que se encuentran en contacto con "gente común" (Graham et al., en prensa, p. 5). En este sentido, las comunicaciones en social media difuminan la distinción entre lo público y lo privado, en donde un mensaje que revela aspectos de la vida privada es hecho público y político (Nusselder, 2013).

La adopción de estilos de comunicación que tienen como objetivo conectar con la "gente común" a través de la asociación con elementos de la cultura popular es conocida como política de la celebridad (Street, 2012; Loader, Vromen y Xenos, 2015). Este tipo de política se da en espacios de comunicación donde los representantes políticos buscan activamente el respaldo y atención de las personas como una audiencia, más que como ciudadanos (Street, 2012). En contraste con el modelo liberal racional de compromiso político, en estos espacios la experiencia de conexión entre ambas partes se apoya en pasiones y sentimientos, los cuales son las fuentes de la afinidad, identificación, evaluación, cuestionamiento o críticas que puedan producirse (Loader et al., 2015). Este aspecto es propiciado por el mismo diseño de las plataformas de social media, el cual alienta la rápida toma de posiciones y actitudes en las discusiones que se suscitan (Nusselder, 2013). De acuerdo con Dean (2016), estas plataformas permiten la expresión de aprobación a través de funcionalidades como likes y follows, provocando $\mathrm{y}$ formando afecto por figuras, lo que funciona como una nueva forma de aclamación. En la aclamación, las características singulares de distintas expresiones individuales se pierden, quedando solo un efecto acumulativo 
en el cual la formación de determinadas reacciones incentiva su propia reproducción.

Por otro lado, estas mismas plataformas también admiten el retiro de la aprobación o incluso la expresión de desaprobación (Dean, 2016). Tyler y Bennett (2010) sugieren que el odio, desprecio o rechazo a personalidades también puede servir como elemento formador de comunidades, como un "anti-fandom". Estas figuras hacen posible reafirmar distinciones sociales y declarar que "yo no soy así" (Tyler y Bennett, 2010, p. 380). De esa manera, funcionan como "malos objetos" ${ }^{3}$, de los cuales las personas se distancian colectivamente, lo que les permite formar y preservar conexiones sociales, al mismo tiempo que mantienen divisiones sociales y patrones de rechazo (Beer, 2017). Este patrón es especialmente notorio en los años recientes, con el surgimiento de personalidades mediáticas polarizantes. De acuerdo con Davies (2017), recientemente se puede observar cómo determinadas personalidades con presencia en social media han ganado relevancia a partir de la emisión de opiniones agresivas o polémicas sobre temas coyunturales. Estas personalidades pueden servir tanto como objetos de aclamación y como "malos objetos", dependiendo de la comunidad a la cual se adhieran los usuarios de social media.

\section{El problema del trol}

Definir claramente el concepto de trol es una tarea compleja debido a los cambios que ha sufrido por su popularización con el paso del tiempo. De acuerdo con Bishop (2013, 2014a, 2014b), el trolling ha pasado de significar "provocar a otros para el entretenimiento de una comunidad virtual" a significar "abusar de otros por goce personal". El momento clave en el cambio entre ambos significados fue la popularización de la imagen de Anonymous y el estilo de trolling que surge de sitios como 4chan, por lo que Bishop hace la distinción entre el estilo de trolling clásico, classic trolling, y el estilo de trolling de Anonymous, Anonymous trolling (2013, 2014a, 2014b). Es precisamente este segundo estilo de trolling el que ha ganado terreno en el espacio común, provocando frecuentemente pánico moral y cobertura negativa por parte de los medios tradicionales (Bishop, 2014b). Con el objetivo de esclarecer la definición de trol, Bishop (2013, 2014a, 2014b) propone una clasificación de doce tipos de usuarios de internet que practican el trolling con motivaciones distintas. Según esta clasificación, el tipo de usuario al que comúnmente se le nombra como trol es un Snert. Un Snert es un hater, alguien a quien le gusta enardecer las situaciones por ningún tipo de beneficio real para otros usuarios. Un Snert practica el

3 Traducción literal de bad object (Tyler y Bennett, 2010, p. 377). 
trolling para dañar a otros por motivaciones antisociales para su propio entretenimiento. Este tipo es distinto de un Troll (con $T$ mayúscula). Los Trolls son eyeballs, observadores que miran lo que otros hacen esperando el momento oportuno para mandar un mensaje provocativo. Motivado por la generación de caos, un Troll practica el trolling para entretener a otros, produciendo tretas que generan diversión a una comunidad de usuarios.

No obstante, a pesar de esta clasificación, el hecho de nombrar a un usuario como trol sigue siendo problemático. A diferencia de Coleman (2010b), quien en cierta medida coincide con Bishop al entender el trolling como una práctica similar a la de un bromista, Bergstrom (2011) sostiene que nombrar a otro usuario como trol también puede ser una forma de justificar el castigo a usuarios acusados de transgredir las normas de una comunidad virtual. Debido a que actualmente el término trol tiene una carga negativa, nombrar a otro usuario de esta manera es una forma de silenciar a los acusados de transgredir, así como de dar por terminado un debate y negarse a reflexionar sobre la posición de uno mismo y de la comunidad a la que uno pertenece (Bergstrom, 2011).
Un desarrollo reciente que complejiza aún más la definición de trol es el surgimiento de troles políticos. Las tácticas en el trolling político van desde provocaciones a oponentes ideológicos para inducir enfrentamientos en secciones de comentarios, hasta esfuerzos coordinados para spamear mensajes y tratar de abrumar a usuarios en plataformas de social media (Sanfilippo, Yang y Fichman, 2017). Reportes de casos en distintos países de Europa del Este señalan la existencia de troles políticos financiados por gobiernos extranjeros -específicamente Rusia- que desean ejercer influencia en el escenario político de otros países (Aro, 2016; Zelenkauskaite y Niezgoda, 2017), así como troles políticos financiados por gobiernos autoritarios que desean atacar a la oposición política (Mihaylov, Koychev, Georgiev y Nakov, 2015; Pearce, 2015). Sin embargo, distinguir entre este tipo de trol político pagado (una práctica similar al astroturfing ${ }^{4}$ ) y un usuario que efectúa el trolling político por un desacuerdo ideológico real ha probado ser una tarea difícil, debido a que la misma práctica del trolling es local y contextual (Sanfilippo et al., 2017). Zelenkauskaite y Niezgoda (2017) encuentran que las acusaciones de ser un trol político pagado

4 El término astroturfing se refiere a la práctica de falsificar el apoyo a una causa, marca o personalidad, que pretende aparentemente ser espontáneo o natural. En el caso de los troles políticos pagados, se trata de mostrar que un partido, gobierno o personalidad tiene apoyo en social media, con usuarios dispuestos a defender, así como a atacar a los oponentes. 
muchas veces son hechas en tono sarcástico o humorístico, e incluso, ocasionalmente, algunos usuarios se autoidentifican irónicamente con esta etiqueta. Estas dos prácticas terminan reforzando la incertidumbre sobre las acusaciones. Más aún, algunas formas de trolling político, motivadas por desacuerdo ideológico, pueden ser vistas como positivas por una comunidad de usuarios que coinciden con la crítica expresada o que la consideran correcta en su forma, al no tratarse de un tipo de trolling perverso hecho solo con la intención de dañar y obtener goce personal (Sanfilippo et al., 2017).

No obstante, a pesar de la incertidumbre sobre la naturaleza de las prácticas de los troles políticos, estas logran tener repercusiones a una escala mayor. Gracias a las características de las plataformas de social media, el material y las discusiones generadas por los troles políticos es visible, esparcible, replicable y persistente en el tiempo (Pearce, 2015). Debido a esto, para algunos de los troles políticos el ataque en sí mismo es más importante que la veracidad de las afirmaciones o el contenido difundido (Pearce, 2015; Aro, 2016). De esta manera, la producción de material sin atribuciones y que puede ser distribuido de manera anónima, como los memes, también es una posible vía de difusión de información sesgada o que ataca a oponentes políticos (Pearce, 2015). Es así como es posible que las tácticas de manipulación en social media, en especial las de troles políticos organizados, repercutan en los medios tradicionales, los cuales, debido a su predilección por el sensacionalismo, su constante necesidad por la novedad y énfasis en rentabilidad económica, se vuelven un vehículo ideal para la difusión de estos contenidos e ideas a una audiencia mayor (Marwick y Lewis, 2017).

\section{Metodología}

El presente artículo es resultado de una investigación cualitativa exploratoria de rasgos etnográficos. La pregunta de investigación que guía esta exploración es la siguiente: ¿qué escenario conforman las interacciones cotidianas en discusiones sobre la política peruana en social media? En este sentido, se trata de observar las prácticas de los individuos en su cotidianidad, insertadas en el contexto en el que ocurren (Bray, 2008). Específicamente, se toman herramientas de lo que Kozinets denomina netnografía (Kozinets, Dolbec y Earley, 2014; Kozinets, en prensa). La netnografía es el estudio comprehensivo de la interacción y experiencia en línea, y se caracteriza por concentrarse en formas de comunicación e información tecnológicamente mediadas y archivadas. Entre los distintos tipos de netnografías presentados por Kozinets (en prensa), se recogen elementos de la netnografía simbólica y la netnografía humanista, dado que se usa información e interacciones de social media para caracterizar a determinados usuarios con el objetivo de atender 
preguntas sobre cuestiones sociales; por ejemplo, de qué manera algunas tecnologías colaboran en la formación de estereotipos, y cómo estas mismas tecnologías forman o transforman las prácticas políticas.

La exploración se realizó en la búsqueda de interacciones entre usuarios de Twitter en donde se utilizan los términos fujitroll, caviar, caviartroll, mudotroll, PPKtroll y apratroll. Abarcó 14781 tuits emitidos en un período de 60 días, del 1 de julio al 30 de agosto del 2017. La selección de este tiempo no responde a algún criterio específico, en tanto esta primera revisión trató de observar las interacciones cotidianas, más allá de su relación con sucesos determinados. En el caso del término caviar, se seleccionaron solo tuits en español que comentaban hechos o personajes de la política peruana, debido a que su uso es diverso por usuarios de distintos países.

Por otro lado, también se examinaron las interacciones alrededor de hashtags y términos relacionados con acontecimientos que afectaron a ministros del gobierno de Pedro Pablo Kuczynski, como \#SaavedraNoSeVa, censura al ministro de Educación Jaime Saavedra (Zubieta, 2016); \#ConfianzaParaThorne, renuncia del ministro de Economía Alfredo Thorne ("Alfredo Thorne tendrá que renunciar...", 2017); y "beca Martens", acusación a la ministra de Educación Marilú Martens de influir en la postulación de su hijo a una beca estatal
(“Ministra Marilú Martens responde a denuncia...", 2017). En esta revisión, se trató de observar las discusiones alrededor de sucesos que involucraban a personalidades específicas debido a la importancia de la tendencia de la personalización en la política actual. Esta exploración abarcó 8924 tuits emitidos en distintos períodos: del 22 de noviembre al 21 de diciembre del 2016 para \#SaavedraNoSeVa, del 21 al 22 de junio del 2017 para \#ConfianzaParaThorne, y del 31 de julio al 24 de agosto del 2017 para "beca Martens". En los tres casos, se trata de los períodos en los cuales se registra la mayor o total cantidad de tuits con los hashtags o términos indicados. La metodología aplicada sigue a estos objetos-términos a través de una red de interacciones; en este sentido, el "lugar de campo" se constituye una vez que el investigador decide prestarle atención a una red de prácticas determinadas (Hine, 2017; Burrell, 2017).

La principal herramienta tomada de la netnografía es la observación no participante de prácticas en línea, también conocida como lurking (Kozinets et al., 2014). El lurking permite aproximarse a lo vernáculo de los medios digitales (Coleman, 2010a) - los materiales o prácticas dependen y son propios de tecnologías digitales, como memes o comentarios en social media - de una manera no intrusiva. Asimismo, también se revisó información -como notas periodísticas, posts en foros, blogs y Facebook- que contribuyera a contextualizar la circulación de significados (Winter, 2014). 


\section{Identidades en contextos polarizados virtuales: Ia actualidad política peruana en social media}

La construcción de identidades a partir de la oposición a movimientos o partidos en el Perú ha sido estudiada desde la ciencia política. De acuerdo con Cyr y Meléndez (2017), los individuos que pertenecen a movimientos basados en antiidentidades tienen predisposiciones en contra de un partido o movimiento en particular. La formación de antiidentidades se da en escenarios donde se ha producido una crisis severa del sistema de partidos políticos debido al surgimiento de outsiders carismáticos con proyectos políticos radicales que lograron el apoyo de un grupo importante de la población. En respuesta a estos movimientos, surgen otros sectores que, por el contrario, nunca apoyarán a este proyecto político. En el contexto peruano, el movimiento y el antimovimiento son el fujimorismo y el antifujimorismo.

El antifujimorismo en internet ha sido abordado desde la perspectiva del ciberactivismo en el estudio del colectivo No a Keiko en la campaña presidencial del 2011 (Lama, 2013). Ya desde ese entonces se hace mención a troles que atacan a antifujimoristas desde perfiles aparentemente falsos, aunque en los testimonios recabados en esa investigación aún no se les llamaba fujitrolls. En la actualidad, el uso de la etiqueta fujitroll es parte de la cotidianidad de las discusiones políticas en social media. El hecho de ser señalado como fujitroll generalmente va acompañado de acusaciones de serlo a cambio de un beneficio económico o material. Estas acusaciones tienen base en la denuncia que se hizo durante la segunda vuelta de la campaña presidencial del 2016, en la cual se indicaba que el partido fujimorista Fuerza Popular contaba con un equipo de troles pagados, quienes se encargaban de crear cuentas y hashtags para apoyar a la candidata Keiko Fujimori, así como de atacar a sus oponentes -los partidarios del entonces candidato Pedro Pablo Kuczynski y los antifujimoristas- de manera tóxica y agresiva (Marchán, 2016). Asimismo, la identidad de algunos de los acusados fue revelada a través de la cuenta de Twitter @fujitrolls (Arellano, 2016). Por otro lado, una frase usada frecuentemente en las acusaciones de ser fujitroll es "toma tu táper" cia a denuncias tanto de las campañas presidenciales del 2011 como del 2016, en las que el fujimorismo fue acusado de repartir alimentos (Jiménez, 2011) e incluso táperes con dinero en efectivo ("Denuncian que partido de Keiko entregó táperes con dinero a

5 Por ejemplo, se pueden ver tuits de usuarios en el siguiente enlace: twitter.com/ search?f=tweets\&q=\%E2\%80\%9Ctoma\%20tu $\% 20 t \% C 3 \% A 1$ per $\% E 2 \% 80 \% 9 D \&$ src $=$ typd 
campesinos", 2016) para conseguir apoyo en actividades proselitistas realizadas en zonas con población en situación de pobreza.

No obstante, una revisión de las interacciones en las que se esgrimen estas acusaciones revela que estas a menudo no sirven para señalar que el oponente político sea realmente un trol político pagado, sino que se utilizan como una forma de descalificación. Asimismo, en ocasiones, la etiqueta de fujitroll no es aplicada solo a quienes son clara y abiertamente fujimoristas, sino también a otros usuarios que critican a los oponentes del fujimorismo - por ejemplo, al gobierno del actual presidente Pedro Pablo Kuczynski-, más allá de si estos mismos usuarios también se oponen al fujimorismo, por ejemplo, usuarios de una tendencia política de izquierda, que se oponen tanto al fujimorismo como al gobierno de Pedro Pablo Kuczynski. Si de por sí la diferenciación entre quienes defienden al fujimorismo y atacan a sus oponentes por motivaciones ideológicas y quienes lo hacen por un beneficio económico es poco clara, el uso indiscriminado de la etiqueta fujitroll entre cualquiera que critique a los oponentes del fujimorismo no hace sino agudizar esta falta de claridad. Aún más, la misma frase "toma tu táper" puede ser interpretada como una frase con connotaciones clasistas, en el sentido de que estereotipa el apoyo político de los sectores populares a cambio de bienes materiales.
En este sentido, podemos observar la operación de dos dinámicas descritas anteriormente. Por una parte, la asignación de la etiqueta fujitroll parece servir, además de para señalar a quienes apoyan y defienden al fujimorismo, como una forma de castigar a usuarios que actúan de una manera distinta a la esperada, similar al estudio de Bergstrom (2011). En este caso, en algunas ocasiones, se le llama fujitroll a quien no coincide totalmente con el antifujimorismo, como movimiento, o con líderes de opinión antifujimoristas. En estas interacciones, nombrar a otro usuario como fujitroll cancela su crítica y anula la posibilidad de reflexionar sobre esta. Por otra parte, la figura del fujitroll parece cumplir la función de un "mal objeto" (Tyler y Bennett, 2010; Beer, 2017). Señalar a otro como fujitroll no solo significa distanciarse de la imagen que el antifujimorismo tiene del seguidor fujimorista - por ejemplo, véase la figura $1-$, sino también mantener y reforzar los lazos colectivos de la identidad del antifujimorismo como movimiento, esto es, el total rechazo al fujimorismo. De esta manera, se dice "nosotros (los antifujimoristas) no somos así (como los fujimoristas)".

Una dinámica similar sucede con la figura del caviar. La palabra caviar fue popularizada en los medios peruanos por comentaristas políticos de derecha como un término peyorativo para sectores progresistas acomodados. No obstante, una revisión de las 
Figura 1. Caricatura del dibujante Cossio que circuló en páginas antifujimoristas durante la campaña del 2016

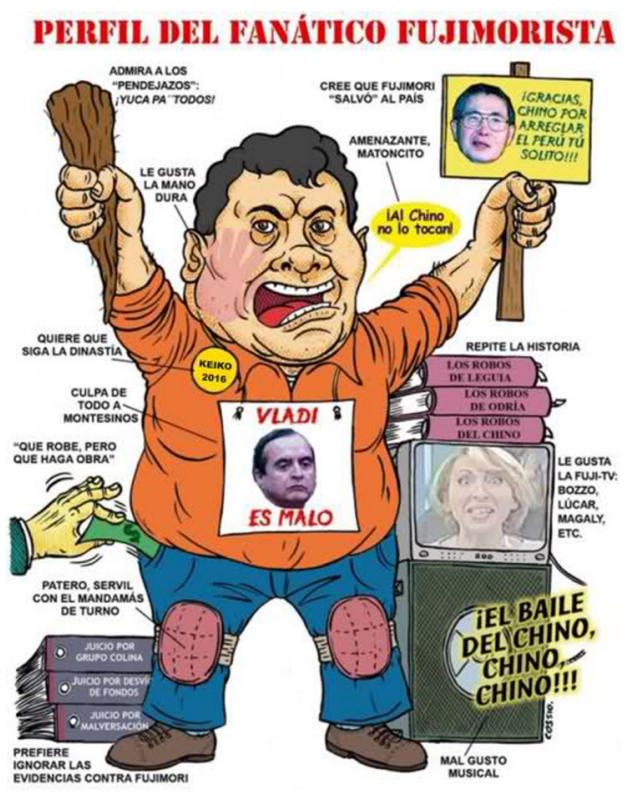

Fuente: Gerónimo (2016)

interacciones cotidianas en social media demuestra que esta etiqueta también ha ganado popularidad en este ámbito. Esto es especialmente notorio en las descripciones de los perfiles de usuarios de derecha, quienes suelen utilizar el término anticaviar - a veces en forma de hashtag, \#anticaviar - para describir su posición política. Quienes se denominan a sí mismos anticaviares utilizan el término caviar - o a veces su variante, caviartroll - para reafirmar su identidad. Al señalar a otro usuario como caviar, lo que se trata de decir es que uno no tiene las características negativas que se le atribuyen al caviar, es decir, el alejamiento de la realidad, la superficialidad y la hipocresía. Asimismo, de manera similar a lo señalado por Davies (2017), algunas figuras mediáticas han logrado posicionarse alrededor de lo que se podría denominar antiprogresismo. Personalidades políticamente conservadoras, por ejemplo, el periodista Phillip Butters, han logrado alcanzar una importante cantidad de seguidores en plataformas como Twitter -más de 350000 seguidores $^{6}$ - y Facebook -más de 250000 likes $^{7}$ - a partir de la emisión de opiniones de carácter agresivo en temas coyunturales polémicos (véase la figura 2). Este tipo de personalidades sirve como objetos de aclamación o "malos objetos", dependiendo de la comunidad, ideología o movimiento político al cual pertenezcan los usuarios.

No obstante, estas prácticas no terminan en el fujimorismo y el antifujimorismo, o con los caviares y anticaviares. Por el contrario, la etiqueta de trol político se ha extendido a los seguidores de otros movimientos y personalidades políticas. Algunos ejemplos son los mudotrolls - usuarios que atacan a oponentes del alcalde de

6 Perfil de Twitter: twitter.com/phill_butters. Cantidad de seguidores a agosto del 2017.

7 Página de Facebook: www.facebook.com/PhillipButtersOficial/. Cantidad de likes a agosto del 2017. 
Figura 2. Video de Phillip Butters emitiendo su opinión en contra de la inclusión del enfoque de género en instituciones estatales

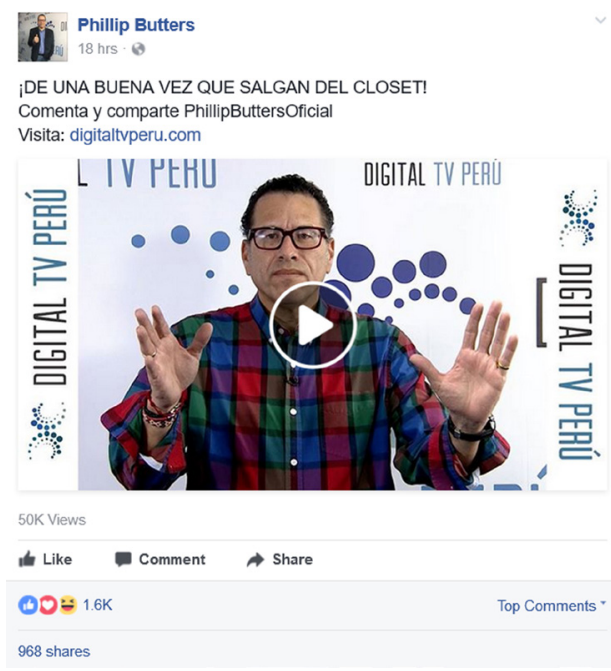

Fuente: Butters (2017)

Lima, Luis Castañeda, conocido como "Mudo"-, los PPKtrolls - usuarios que atacan a oponentes del gobierno de Pedro Pablo Kuczynski-, o apratrolls - usuarios que atacan a oponentes del partido aprista-. De manera similar a los fujitrolls, en algunos de estos casos, existen acusaciones de troles contratados. En el caso de los mudotrolls, se denunció que distintos usuarios de Twitter que atacan a regidores de la oposición de Castañeda, de hecho, son funcionarios y empleados de la Municipalidad de Lima (Núñez, 2016). En el caso de los PPKtrolls, existen acusaciones de gasto del gobierno en una agencia publicitaria para el manejo de social media (Ramírez, 2017). Si bien en este último caso, hasta el momento, no existen evidencias claras de troles políticos pagados, sino de gastos que podrían considerarse para comunicación y publicidad estatal, este detalle parece no ser relevante para los usuarios y troles opositores del gobierno, quienes han nombrado a esta agencia como trollcenter $^{8}$. A esto se suma el surgimiento de nuevos medios alternativos parcializados, como la página de noticias Manifiesto, que ha ganado cierta relevancia entre personas de derecha que se oponen al gobierno de Kuczynski, especialmente fujimoristas. Manifiesto tiene una línea informativa marcadamente contraria al gobierno, llegando incluso a difundir noticias con titulares engañosos o contenido sin confirmar (véase, por ejemplo, la figura 3).

De esta manera, lo que afirman Garret et al. (2016) parece ser cierto: más allá de las interacciones que los opositores de derecha del gobierno puedan tener con versiones oficiales del gobierno o partidarios de Kuczynski, el hecho de contar con medios que produzcan versiones que apoyan sus creencias sobre sus oponentes políticos e ideológicos les basta para reafirmarse en ellas, descartando las versiones oficiales o con

8 Por ejemplo, se pueden ver tuits de usuarios en el siguiente enlace: twitter.com/search? $\mathrm{f}=\mathrm{t}$ weets\&vertical $=$ default\&q=trollcenter $\% 20$ ppk\&src=typd 
Figura 3. Titular de noticia de la página de noticias Manifiesto

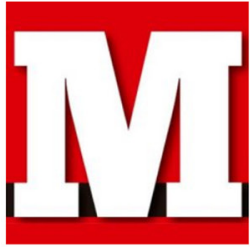

Manifiesto

La prensa escrita jamás será vencida

Buscar.

ENTRADAS RECIENTES

ODEBRECHT QUIERE HACER FAENÓN CON VENTA DE SUS ACCIONES EN PROYECTO OLMOS

Fuente: Rojas (2017)

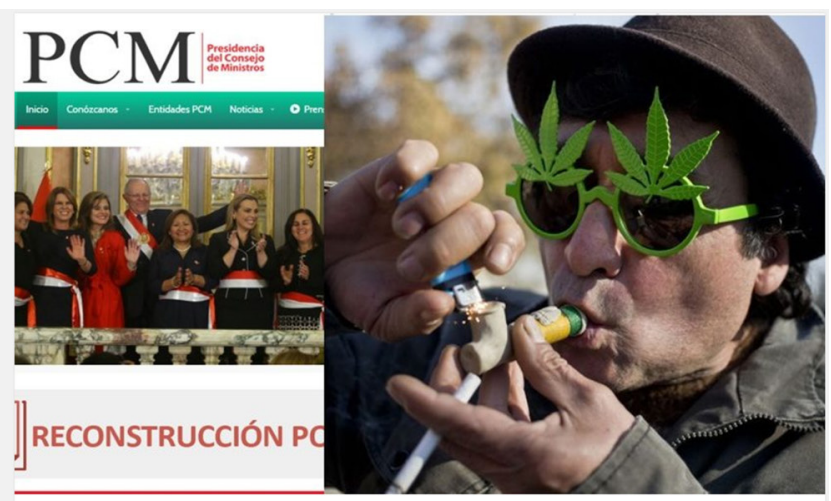

\section{PEPEKAUSA HERESI DENUNCIA QUE DROGADICTOS MANEJAN IMAGEN DEL GOBIERNO}

mayor sustento a las que puedan estar expuestos. En este sentido, lo que importa es el mismo hecho de atacar más que la veracidad de la información que se usa, debido a que quienes son políticamente afines a esta información se encargarán de diseminarla (Pearce, 2015). Es así como el escenario que emerge es similar al descrito por Del Vicario et al. (2016) y por Bessi et al. (2016): comunidades de identidades políticas que pueden interactuar entre sí, pero que se encuentran polarizadas.

\section{Conclusión}

En este artículo, se ofrece un bosquejo de la cotidianidad de las discusiones políticas que ocurren en plataformas de social media en el Perú. A partir de la descripción de tres tendencias actuales en estas interacciones -la polarización en el debate político, la concentración en personalidades y objetos, y las prácticas de los troles-, se ha dado una primera mirada a cómo es que se forman las identidades en las interacciones cotidianas de los usuarios. Si bien las identidades e interacciones políticas en social media en el Perú es un tema que ha sido estudiado anteriormente (Ferrándiz, Ibáñez y Espinosa, 2011; Guevara, 2012), por lo general, la atención se ha centrado en contextos electorales. La motivación detrás de este trabajo es el deseo por llamar la atención sobre la cotidianidad política en social media como un objeto de estudio, en lugar de descartarla como un contexto negativo o superfluo de antemano. 
Debido a la relación cada vez más simbiótica entre social media y los medios tradicionales, en la cual los medios establecidos le dan mayor cabida a información y opiniones políticas emitidas en plataformas como Facebook o Twitter -incluyendo versiones agresivas y manipuladas (Marwick y Lewis, 2017)-, es clave prestar atención a los espacios virtuales y las interacciones que suceden en estos. Existen trabajos de carácter descriptivo sobre cómo los periodistas peruanos recogen información de social media (Espinoza, 2012; Pichihua, 2012, 2017; Vílchez, 2015); no obstante, una mayor atención crítica sobre este nuevo escenario es necesaria.

$\mathrm{Si}$ bien este trabajo representa un esfuerzo exploratorio, a partir de la discusión teórica y la aplicación de conceptos al escenario peruano, se pueden extraer algunas conclusiones y recomendaciones generales. En primer lugar, los periodistas de medios establecidos deben ser conscientes de su responsabilidad al darle visibilidad a contenido que recogen de social media. $\mathrm{Al}$ tratarse de espacios donde impera la polarización y la incertidumbre, su credibilidad y, aún más importante, el mantenimiento de un discurso cívico están en juego en cada momento. En segundo lugar, se debe tener más cuidado con la asignación de la etiqueta de trol a otros usuarios. Si bien resulta imposible controlar a un nivel micro cómo es que los usuarios usan el calificativo de trol, personas como autoridades públicas y académicos que participan en social media deberían ser cuidadosos al calificar a otros usuarios como troles, debido a que sus afirmaciones tienen peso y relevancia para las instituciones que representan y los medios establecidos. Por último, se espera que el presente artículo abra líneas de trabajo sobre la cotidianidad política de social media. En un escenario polarizado, es necesario tratar de conocer al Otro. Trabajos de corte etnográfico, que exploren cuáles son las prácticas de estas comunidades virtuales que frecuentemente son marginadas como objeto de estudio, pero que tienen ideas que llegan al debate público, serían especialmente valiosos.

\section{Referencias}

Alfredo Thorne tendrá que renunciar: Congreso no le renovó confianza. (21 de junio del 2017). El Comercio. Recuperado de elcomercio.pe/poli tica/alfredo-thorne-tendra-renun ciar-congreso-le-renovo-confianza $-436319$

Arellano, J. (12 de mayo del 2016). La cacería de Fujitrolls ha empezado [mensaje en un blog]. Recuperado de arellanojuan.com/la-caceria-defujitrolls-ha-empezado/

Aro, J. (2016). The Cyberspace War: Propaganda and Trolling as Warfare Tools. European View, 15(1), 121-132. doi: 10.1007/s12290-016-0395-5

Barberá, P., Jost, J. T., Nagler, J., Tucker, J. A., y Bonneau, R. (2015). Tweeting from Left to Right: Is Online Political Communication 
More Than an Echo Chamber? Psychological Science, 26(10), 1-12. doi: 10.1177/0956797615594620

Beer,D. (19dejuliodel2017). TheBackfire Effect, Bad Objects, and Changing Our Minds Online. openDemocracy. Recuperado de www.opendemocra cy.net/digitaliberties/david-beer/ backfire-effect-bad-objects-andchanging-our-minds-online

Bergstrom, K. (2011). “Don't Feed the Troll": Shutting Down Debate about Community Expectations on Reddit.com. First Monday, 16(8). Recuperado de firstmonday.org/ojs/ index.php/f m/article/view/34 98/3029

Bessi, A., Zollo, F., Del Vicario, M., Puliga, M., Scala, A., Caldarelli, G., Uzzi, B., y Quattrociocchi, W. (10 de abril del 2016). Users Polarization on Facebook and Youtube. Recuperado de la base de datos arXiv.

Bishop, J. (2013). The Effect of De-Individuation of the Internet Troller on Criminal Procedure Implementation: An Interview with a Hater. International Journal of Cyber Criminology, 7(1), 28-48.

Bishop, J. (2014a). Trolling for the Lulz? Using Media Theory to Understand Transgressive Humour and Other Internet Trolling in Online Communities. En Transforming Politics and Policy in the Digital Age (pp. 155-172). Pensilvania: IGI Global. doi: 10.4018/978-1-4666-60380.ch011
Bishop, J. (2014b). Representations of "Trolls" in Mass Media Communication: A Review of Media-Texts and Moral Panics relating to "Internet Trolling". International Journal of Web Based Communities, 14(1), 7-24.

Bray, Z. (2008). Ethnographic Approaches. En D. Della Porta y M. Keating (Eds.), Approaches and Methodologies in the Social Sciences (pp. 296-315). Nueva York, NY: Cambridge.

Burrell, J. (2017). The Fieldsite as a Network. En L. Hjorth, H. Horst, A. Galloway, y G. Bell (Eds.), The Routledge Companion to Digital Ethnography (pp. 50-60). Nueva York, NY: Routledge.

Butters, Ph. (30 de agosto del 2017). ¡De una buena vez que salgan del clóset! [archivo de video]. Recuperado de https://www.facebook.com/Phillip ButtersOficial/videos/1968254 540112655/

Coleman, G. (2010a). Ethnographic Approaches to DigitalMedia. Annual Review of Anthropology, 39, 487-505. doi: 10.1146/annurev.anthro.012809. 104945

Coleman, G. (2010b). Hacker and Troller as Trickster. Interprete. Recuperado de gabriellacoleman. org/blog/?p=1902

Cyr, J., y Meléndez, C. (9 de mayo del 2017). Anti-Identity Movements in Latin America: Anti-chavismo, Antifujimorismo, and Anti-uribismo 
in Comparative Perspective [mensaje en un blog]. Recuperado de blog. mpsanet.org/2017/05/09/anti-identitymovements-in-latin-america/

Davies, W. (2017). The New Digital Elites: Neoliberalism, Technocrats, and their Failures [exposición en la Universidad de Goldsmiths, Londres, archivo de video]. Recuperado de www.youtube.com/watch? $v=\mathrm{vdm}$ 7E_zZnN4

Dean, M. (2016). Political Acclamation, Social Media and the Public Mood. European Journal of Social Theory, 20(3), 1-20. doi: 10.1177/ 1368431016645589

Della Porta, D. (2011). Communication in Movement: Social Movements as Agents of Participatory Democracy. Information, Communication \& Society, 14(6), 800-819. doi: 10.1080/1369118X .2011 .560954

Del Vicario, M., Zollo, F., Caldarelli, G., Scala, A., y Quattrociocchi, W. (21 de octubre del 2016). The Anatomy of Brexit Debate on Facebook. Recuperado de la base de datos arXiv.

Denuncian que partido de Keiko entregó táperes con dinero a campesinos. (3 de junio del 2016). RPP Noticias. Recuperado de rpp. pe/peru/puno/denuncian-quefuerza-popular-entrego-tapers-condinero-a-campesinos-noticia-968159

Espinoza, A. A. (2012). La cultura digital en el periodismo impreso. El caso del diario El Comercio (tesis de licenciatura). Recuperado de http:// tesis.pucp.edu.pe/repositorio/ handle/123456789/1662

Ferrándiz, J., Ibáñez, C., y Espinosa, A. (2011). Racismo 2.0: expresiones de prejuicio en las redes sociales virtuales tras las elecciones generales de 2011. Politai, 2(3), 75-83.

Garret, R. K., Weeks, B. E., y Neo, R. L. (2016). Driving a Wedge between Evidence and Beliefs: How Online Ideological News Exposure Promotes Political Misperceptions. Journal of Computer-Mediated Communication, 21(5), 331-348. doi: 10.1111/ jcc4.12164

Gerónimo, J. [@jgpl1770]. (4 de abril del 2016). Perfil del fanático fujimorista [tuit en respuesta a @colchadogui] [imagen]. Recuperado de https:// twitter.com/jgpl1710/status/717097 148119171073

Graham, T., Jackson, D., y Broersma, M. (en prensa). The Personal in the Political on Twitter: Towards a Typology of Politicians' Personalized Tweeting Behaviours. En J. Schwanholz, T. S. Graham, y P. Stoll (Eds.), Managing Democracy in the Digital Age: Internet Regulation, Social Media Use, and Online Civic Engagement. Berlín: Springer.

Guevara, L. A. (2012). Estereotipos, emociones y tendencia de acción intergrupal en Facebook durante las elecciones generales del 2011 (tesis de licenciatura). Recuperado de http:// tesis.pucp.edu.pe/repositorio/ handle/123456789/4424 
Hart, P. S., y Nisbet, E. C. (2011). Boomerang Effects in Science Communication: How Motivated Reasoning and Identity Cues Amplify Opinion Polarization About ClimateMitigation Policies. Communication Research, 39(6), 1-23. doi: 10.1177/0093650211416646

Hermans, L., y Vergeer, M. (2012). Personalization in E-Campaigning: A Crossnational Comparison of Personalization Strategies Used on Candidate Websites of 17 Countries in EP Elections 2009. New Media \& Society, 15(1), 72-92. doi: $10.1177 / 1461444812457333$

Hine, C. (2017). From Virtual Ethnography to the Embedded, Embodied, Everyday Internet. En L. Hjorth, H. Horst, A. Galloway, y G. Bell (Eds.), The Routledge Companion to Digital Ethnography (pp. 21-28). Nueva York, NY: Routledge.

Hutchens, M. J., Cicchirillo, V. J., y Hmielowski, J. D. (2015). How Could You Think That?!?! Understanding Intentions to Engage in Political Flaming. New Media $\mathcal{E}$ Society, 17(8), 1201-1219. doi: $10.1177 / 1461444814522947$

Jenkins, H. (2008). Convergence Culture. La cultura de la convergencia de los medios de comunicación. Barcelona: Paidós.

Jensen, M. J., Danziger, J. N., y Venkatesh, A. (2007). Civil Society and Cyber Society: The Role of the Internet in Community Associations and Democratic Politics. The Information Society, 23(1), 39-50. doi: 10.1080/01972240601057528

Jiménez, B. (6 de mayo del 2011). Keiko Fujimori reparte comida entre los pobres a cambio de votos. El Mundo. Recuperado de www. elmundo.es/america/2011/05/06/ noticias/1304691775.html

Kozinets, R. (en prensa). Management Netnography: The Art and Science of Online Cultural Business Research. En C. Cassell, A. Cunliffe, y G. Grandy (Eds.), The SAGE Handbook of Qualitative Business and Management Research Methods. Londres: SAGE.

Kozinets, R., Dolbec, P., y Earley, A. (2014). Netnographic Analysis: Understanding Culture through Social Media Data. En U. Flick (Ed.), The SAGE Handbook of Qualitative Data Analysis (pp. 262-276). Londres: SAGE.

Lama, C. V. (2013). Estrategia comunicacional desarrollada en Facebook por el movimiento social ciberactivista No a Keiko para integrar el activismo online $y$ offline con el fin de impedir la elección presidencial de la candidata Keiko Fujimori en el 2011 (tesis de licenciatura). Recuperado de http:// tesis.pucp.edu.pe/repositorio/ bitstream/handle/123456789/5071/ LAMA_FLORES_CYNTHIA_ ESTRATEGIA_F U J IMORI. pdf?sequence $=1 \&$ isAllowed $=y$ 
Loader, B. D., Vromen, A., y Xenos, M. A. (2015). Performing for the Young Networked Citizen? Celebrity Politics, Social Networking and the Political Engagement of Young People.Media, Culture ESociety,38(3), 1-20. doi: 10.1177/0163443715608261

Marchán, E. (6 de mayo del 2016). Caca con ventilador. Hildebrandt en sus Trece. Recuperado de www. tarata21.com/2016/05/hildebrandt -en-sus-trece-caca-con.html

Marwick, A., y Lewis, R. (2017). Media Manipulation and Disinformation Online. Recuperado de datasociety. net/output/media-manipulationand-disinfo-online/

McGregor, S. C., Lawrence, R. G., y Cardona, A. (2016). Personalization, Gender, and Social Media: Gubernatorial Candidates' Social Media Strategies. Information, Communication \& Society, 20(2), 264-283. doi: 10.1080/1369118X.2016.1167228

Meléndez, C. (4 de setiembre del 2012). Caviares [mensaje en un blog]. Recuperado dejorobadonotredame. blogspot.pe/2012/09/caviares.html

Mihaylov, T., Koychev, I., Georgiev, G. D., y Nakov, P. (2015). Exposing Paid Opinion Trolls. Proceedings of Recent Advances in Natural Language Processing. Recuperado de aclweb. org/anthology/R15-1058

Ministra Marilú Martens responde a denuncia sobre beca del Estado para su hijo. (31 de julio del 2017). Perú21. Recuperado de peru21.pe/ lima/ministra-marilu-martensresponde-denuncia-beca-hijo-91862 Núñez, H. (11 de setiembre del 2016). Los Trolls de la Solidaridad [artículo de Facebook]. Recuperado de www.facebook.com/notes/hern \% C 3\% A 1 n-n \% C 3\%B A \% C 3\% B1ez/los-trolls-de-la-solidaridad /682914238529788

Nusselder, A. (2013). Twitter and the Personalization of Politics. Psychoanalysis, Culture \& Society, 18(1), 91-100.

Nyhan, B., y Reifler, J. (2010). When Corrections Fail: The Persistence of Political Misperceptions. Political Behavior, 32(2), 303-330. doi: 10.1007/ s11109-010-9112-2

Pariser, E. (2011). The Filter Bubble: What the Internet Is Hiding from You. Nueva York, NY: Penguin Press.

Pearce, K. E. (2015). Democratizing Kompromat: The Affordances of Social Media for Statesponsored Harassment. Information, Сommunication \& Society, 18(10), 1158-1174. doi: 10.1080/1369118X.2015.1021705

Pichihua, S. P. (2012). Producción de noticias en las redacciones digitales de Perú21 y Depor (tesis de licenciatura). Recuperado de http:// tesis.pucp.edu.pe/repositorio/ handle/123456789/1388

Pichihua, S. P. (2017). Cómo los periodistas de las secciones Deportes y Política de la Agencia Andina utilizan Twitter como fuente de información para noticias de último minuto (tesis 
de maestría). Recuperado de http:// tesis.pucp.edu.pe/repositorio/ handle/123456789/8357

Ramírez, M. (29 de agosto del 2017). Las pugnas y los nuevos "gurús" de Palacio de Gobierno. Trome. Recuperado de trome.pe/actualidad /pugnas-nuevos-gurus-palaciogobierno-historias-contadas-60431

Rojas, C. (31 de julio del 2017). Pepekausa Heresi denuncia que drogadictos manejan imagen del gobierno. Manifiesto. Recuperadodehttp://manifiesto. net.pe/2017/07/31/pepekausa-heresidenuncia-que-drogadictos-manejanimagen-del-gobierno/

Sanfilippo, M. R., Yang, S., y Fichman, P. (2017). Managing Online Trolling: From Deviant to Social and Political Trolls. Proceedings of the 50th Hawaii International Conference on System Sciences (pp. 1802-1811). doi: 10.24251/HICSS.2017.219.

Shah, D. V., McLeod D. M., Rojas, H., Cho, J., Wagner, M. W., y Friedland, L. A. (2017). Revising the Communication Mediation Model for a New Political Communication Ecology. Human Communication Research, 43(3), 1-14. doi: 10.1111/hcre.12115

Strandberg, K., Himmelroos, S., y Grönlund, K. (2017). Do Discussions in Like-Minded Groups Necessarily Lead to More Extreme Opinions? Deliberative Democracy and Group Polarization. International Political Science Review, 1-17. doi: 10.1177/01925 12117692136
Street, J. (2012). Do Celebrity Politics and Celebrity Politicians Matter? British Journal of Politics \& International Relations, 14(3), 46-356. doi: 10.1111/j.1467-856X.2011.00480.x

Tyler, I., y Bennett, B. (2010). “Celebrity Chav": Fame, Femininity, and Social Class. European Journal of Cultural Studies, 13(3), 375-393. doi: 10.1177/1367549410363203

Van Aelst, P., Sheafer, T., y Stanyer, J. (2012). The Personalization of Mediated Political Communication: A Review of Concepts, Operationalizations, and Key Findings. Journalism, 13(2), 203-220. doi: $10.1177 / 1464884911427802$

Vílchez, P. G. (2015). Convergencia periodística en el Perú. El caso "El Comercio" (tesis de maestría). Recuperado de http://tesis.pucp.edu.pe/repositorio/ bitstream/handle/123456789/6262/ VILCHEZ_SAMANEZ_PRISEA _CONVERGENCIA_PERIODISTI CA.pdf?sequence $=1$

Villanueva, E. (2015). Acción conectiva, acción colectiva y medios digitales: posibilidades para la comunicación política en los tiempos de Internet. Contratexto, 24, 57-76.

Winter, R. (2014). Cultural Studies. En U. Flick (Ed.), The SAGE Handbook of Qualitative Data Analysis (pp. 247261). Londres: SAGE.

Zelenkauskaite, A., y Niezgoda, B. (2017). "Stop Kremlin Trolls": Ideological Trolling as Calling Out, Rebuttal, and Reactions on Online 
News Portal Commenting. First Monday,5(1).Recuperadodefirstmon day.org/ojs/index.php/fm/article/ view/7795/6225

Zubieta, R. (15 de diciembre del 2016). Jaime Saavedra y el camino que lo llevó hasta la censura. El Comercio. Recuperado de elcomercio.pe/politi ca/actualidad/jaime-saavedracamino-llevo-censura-400610 